\title{
De expressões faciais à experiência do jogador: desafios da adaptação automática de jogos sérios com Computação Afetiva
}

\author{
Renan Vinicius Aranha \\ renan.ufg@gmail.com \\ Escola Politécnica - Universidade de São Paulo \\ São Paulo, São Paulo, Brasil
}

\section{RESUMO}

Jogos sérios mesclam os desafios de aplicações de entretenimento e produtividade no oferecimento de uma boa experiência ao usuário. Uma abordagem promissora é a adaptação automática dos jogos em momentos específicos, quando detecta-se que o usuário não está desfrutando de uma boa experiência. Visando a contribuir com este cenário, esta pesquisa de doutorado propõe o desenvolvimento de um framework que possibilite a adaptação automática de jogos sérios em conformidade com o estado emocional e a personalidade do jogador. Para tal finalidade, além do desenvolvimento do arcabouço computacional, encontram-se desafios inerentes ao tratamento de fatores humanos complexos e subjetivos, como as emoções, a personalidade e a experiência do usuário. Neste escopo, a avaliação da experiência do jogador por meio da análise de expressões faciais apresenta-se como um desafio. No presente estudo foi verificada uma baixa correlação entre expressões faciais e o engajamento do jogador. Adicionalmente, emoções negativas não necessariamente representam uma experiência ruim com o jogo, tornando esta proposta ainda mais complexa.

\section{CCS CONCEPTS}

- Human-centered computing $\rightarrow$ User studies; • Social and professional topics $\rightarrow$ User characteristics.

\section{KEYWORDS}

Computação Afetiva, Jogos Sérios, Experiência do Jogador

\section{INTRODUÇÃO E MOTIVAÇÃO}

Jogos sérios, aplicações criadas com o intuito de unir o potencial motivador dos jogos com a realização de atividades que visam a oportunizar aprendizado e/ou desenvolvimento de habilidades, mesclam os desafios das aplicações de produtividade e de entretenimento no sentido de oferecer uma boa experiência ao usuário. Embora visem a oferecer certo grau de diversão, as características complexas das atividades permanecem [10]. A adaptação automática destas aplicações pode contribuir para o aprimoramento do processo de interação, oferecendo intervenções quando o usuário não estiver vivenciando uma boa experiência com o software [10,12].

Todavia, a avaliação da experiência do jogador, que inclui conceitos como imersão e engajamento, é um processo ainda complexo, uma vez que as abordagens majoritariamente utilizadas para tal finalidade podem interromper a interação ou envolver análises de seres humanos, tornando o processo manual [9]. Uma abordagem promissora neste escopo de avaliação automática consiste na adoção de técnicas de Computação Afetiva, em especial o reconhecimento de expressões faciais associadas a emoções por meio do processamento de imagens [5].

\author{
Fátima L. S. Nunes \\ fatima.nunes@usp.br \\ Escola Politécnica - Universidade de São Paulo \\ São Paulo, São Paulo, Brasil
}

Assim, esta pesquisa de doutorado tem como objetivo desenvolver um framework que possibilite a adaptação de jogos sérios considerando tanto as expressões faciais quanto as características individuais do usuário, mais especificamente de seus traços de personalidade. Para além dos desafios de desenvolvimento do arcabouço computacional, já relatados anteriormente [1], este resumo enfoca um principais desafios desta pesquisa: a relação entre as expressões faciais e a experiência do jogador.

\section{TRABALHOS RELACIONADOS}

Embora a análise de expressões faciais possa oferecer importantes indicadores, como a probabilidade de ocorrência de uma entre as seis emoções universais (alegria, medo, nojo, raiva, tristeza e surpresa), ainda não é evidente o papel de cada emoção no processo de interação com jogos. Birk et al. [7] and Bopp et al. [8], por exemplo, ressaltam que mesmo emoções consideradas negativas (como raiva, por exemplo) podem estar associadas a uma boa experiência. Portanto, a associação entre emoções positivas e uma boa experiência não é necessariamente adequada.

Em complemento, uma revisão de literatura conduzida por Robinson et al. [11] revela que o campo de jogos afetivos ainda encontra-se em estágio inicial. Adicionalmente, tais aplicações adotam majoritariamente técnicas que fornecem uma diferente compreensão dos fatores emocionais. Portanto, investigações acerca da avaliação da experiência considerando a análise de expressões faciais ainda é um tópico a ser explorado.

\section{DEFINIÇÃO DA PROPOSTA}

Para propiciar a adaptação automática de jogos sérios conforme o estado emocional e os traços de personalidade do jogador, esta pesquisa prevê o aprimoramento do framework Easy Affecta. Proposto em [2], o framework é inicialmente composto por: i) um módulo de reconhecimento de expressões faciais; ii) um módulo para configuração, pelo instrutor, das regras de adaptação a serem aplicadas conforme os estados emocionais dos pacientes; iii) um módulo de adaptação, que aplica ao jogo as regras de adaptação.

A principal contribuição desta pesquisa, que acarretará no aprimoramento do framework, consiste na definição e implementação de estratégias para a definição das adaptações de modo automático. Assim, minimiza-se a necessidade de um agente intermediário para a configuração dos perfis de usuário, papel então exercido pelo fisioterapeuta na primeira versão do EasyAffecta. Este processo envolve: i) a elaboração de uma abordagem que possibilite a avaliação da experiência do jogador; ii) o desenvolvimento de um módulo para definir adaptações considerando os traços de personalidade e o estado emocional dos usuários; iii) investigações sobre estratégias que possibilitem o relacionamento entre características do usuário e os 
elementos adaptáveis em software. Atualmente, o foco encontra-se na avaliação da experiência do jogador.

\section{RESULTADOS PRELIMINARES}

Como resultados preliminares, além da implementação de considerável parte do arcabouço proposto, destacam-se publicações sobre: os impactos dos traços de personalidade na experiência dos usuários de Realidade Virtual [6]; a adaptação de software conforme o estado emocional do usuário [5]; o desenvolvimento de uma análise comparativa acerca de ferramentas de código aberto para o reconhecimento de expressões faciais [3]; a relação entre expressões faciais, interações e engajamento do jogador [4].
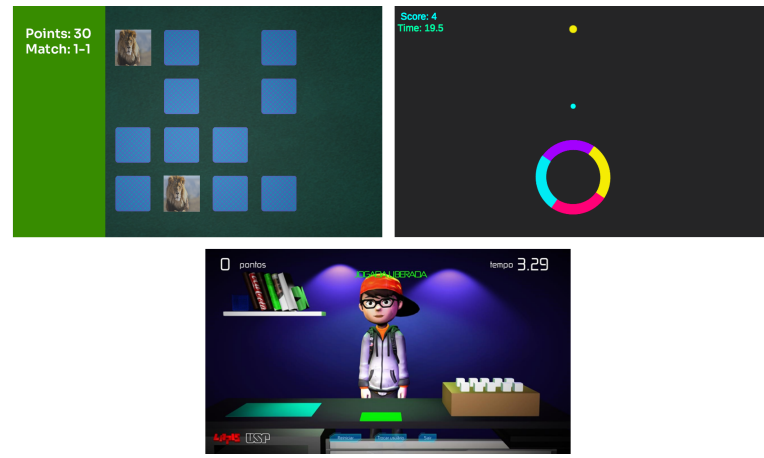

Figura 1: Jogos utilizados no estudo.

No tocante à relação entre as expressões faciais associadas a emoções discretas e a experiência do jogador [4], uma análise comparativa foi elaborada visando a identificar se existe correlação entre tais variáveis. A Tabela 1 apresenta os principais resultados obtidos no estudo, que considerou três jogos com diferentes características (ilustrados pela Figura 1), bem como diferentes abordagens de avaliação do engajamento. Como pode-se observar, os índices encontrados são, majoritariamente, correlações fracas.

Tabela 1: Índices de correlação entre expressões faciais de emoções discretas e engajamento.

\begin{tabular}{llll}
\hline Emoção & Caso 1 & Caso 2 & Caso 3 \\
\hline Alegria & 0.6 & -0.05 & 0.22 \\
Medo & 0.08 & 0.15 & -0.28 \\
Nojo & 0.19 & 0.18 & 0 \\
Raiva & 0.09 & -0.25 & -0.03 \\
Tristeza & 0.06 & 0.06 & 0 \\
Surpresa & 0.32 & 0.04 & -0.12 \\
Neutro & - & -0.02 & 0 \\
Desprezo & -0.05 & - & - \\
\hline
\end{tabular}

\section{CONSIDERAÇÕES FINAIS}

A adaptação automática de jogos, em especial em decorrência da análise de fatores humanos, como emoção e personalidade, é um campo de pesquisa promissor, porém envolto em inúmeros desafios. Os resultados preliminares indicam que correlações entre tais variáveis podem existir, porém geralmente de modo fraco e variando conforme o jogo adotado. Estes resultados revelam ainda a necessidade de se desenvolver mais investigações sobre como as expressões faciais podem indicar a experiência do jogador. Portanto, as próximas etapas do projeto compreendem investigações acerca de outras técnicas, como a algoritmos de aprendizado de máquina, para o processamento destes dados visando a avaliação da experiência do jogador.

\section{AGRADECIMENTOS}

Este trabalho foi realizado com apoio de: Coordenação de Aperfeiçoamento de Pessoal de Nível Superior - Brasil (CAPES) - Código de Financiamento 001; Conselho Nacional de Desenvolvimento Cientifico e Tecnológico (CNPq) (Processo 157535/2017-7); Fundação de Amparo à Pesquisa do Estado de São Paulo (FAPESP) (Processos 14/50889-7): Instituto Nacional de Ciência e Tecnologia em Medicina Assistida por Computação Científica (INCT-MACC).

\section{REFERÊNCIAS}

[1] Renan Aranha and Fátima Nunes. 2020. Um framework para a adaptação de jogos sérios com Computação Afetiva e Computação de Personalidade. In Anais Estendidos do XXII Simpósio de Realidade Virtual e Aumentada (Evento Online). SBC, Porto Alegre, RS, Brasil, 7-8. https://doi.org/10.5753/ svr_estendido.2020.12944

[2] Renan Vinicius Aranha. [n.d.]. EasyAffecta: um framework baseado em Computação Afetiva para adaptação automática de jogos sérios para reabilitação motora. https://doi.org/10.11606/d.100.2017.tde-24072017-083504

[3] Renan Vinicius Aranha, André Biondi Casaes, and Fátima L. S. Nunes. 2020. Influence of environmental conditions in the performance of open-source software for facial expression recognition. In Proceedings of the 19th Brazilian Symposium on Human Factors in Computing Systems. ACM. https://doi.org/ $10.1145 / 3424953.3426630$

[4] Renan Vinicius Aranha, Leonardo Nogueira Cordeiro, Lucas Mendes Sales, and Fátima L. S. Nunes. 2021. Engagement and discrete emotions in game scenario: is there a relation among them?. In Proceedings of the IFIP TC13 International Conference on Human-Computer Interaction. IFIP.

[5] Renan Vinicius Aranha, Cleber Gimenez Correa, and Fatima L. S. Nunes. 2019. Adapting software with Affective Computing: a systematic review. IEEE Transactions on Affective Computing (2019), 1-1. https://doi.org/10. $\overline{1109 /}$ taffc.2019.2902379

[6] Renan Vinicius Aranha, Ricardo Nakamura, Romero Tori, and Fatima L. S. Nunes. 2018. Personality Traits Impacts in Virtual Reality's User Experience. In 2018 20th Symposium on Virtual and Augmented Reality (SVR). IEEE. https://doi.org/ 10.1109/svr.2018.00019

[7] Max V. Birk, Ioanna Iacovides, Daniel Johnson, and Regan L. Mandryk. 2015. The False Dichotomy between Positive and Negative Affect in Game Play. In Proceedings of the 2015 Annual Symposium on Computer-Human Interaction in Play (London, United Kingdom) (CHI PLAY '15). Association for Computing Machinery, New York, NY, USA, 799-804. https://doi.org/10.1145/2793107.2810258

[8] Julia Ayumi Bopp, Elisa D. Mekler, and Klaus Opwis. 2016. Negative Emotion, Positive Experience? Emotionally Moving Moments in Digital Games. Association for Computing Machinery, New York, NY, USA, 2996-3006. https: //doi.org/10.1145/2858036.2858227

[9] João Bosco Borges, Carmen Li Juy, Izac Sidarta De Andrade Matos, Paulo Vitor Angelo Silveira, and Ticianne De Gois Ribeiro Darin. 2020. Player Experience Evaluation: Which instrument should I use? Journal on Interactive Systems 11, 1 (Dec. 2020), 74-91. https://doi.org/10.5753/jis.2020.765

[10] Stefan Göbel and Viktor Wendel. 2016. Personalization and Adaptation. Springer International Publishing, Cham, 161-210. https://doi.org/10.1007/978-3-31940612-1_7

[11] Raquel Robinson, Katelyn Wiley, Amir Rezaeivahdati, Madison Klarkowski, and Regan L. Mandryk. 2020. "Let’s Get Physiological, Physiological!": A Systematic Review of Affective Gaming. In Proceedings of the Annual Symposium on Computer-Human Interaction in Play (Virtual Event, Canada) (CHI PLAY '20). Association for Computing Machinery, New York, NY, USA, 132-147. https: //doi.org/10.1145/3410404.3414227

[12] R. Tadayon, A. Amresh, T. McDaniel, and S. Panchanathan. 2018. Real-time stealth intervention for motor learning using player flow-state. In 2018 IEEE 6th International Conference on Serious Games and Applications for Health (SeGAH). 1-8. 